Revue d'histoire de l'Amérique française

REVUE D.HISTOIRE DE L'AMÉRIQUE FRANÇAISE

\title{
Un plagiaire illustre : François Perrin du Lac
}

\section{Fernand Grenier}

Volume 7, numéro 2, septembre 1953

URI : https://id.erudit.org/iderudit/301592ar

DOI : https://doi.org/10.7202/301592ar

Aller au sommaire du numéro

Éditeur(s)

Institut d'histoire de l'Amérique française

ISSN

0035-2357 (imprimé)

1492-1383 (numérique)

Découvrir la revue

Citer cet article

Grenier, F. (1953). Un plagiaire illustre : François Perrin du Lac. Revue d'histoire de l'Amérique française, 7(2), 207-223. https://doi.org/10.7202/301592ar d'utilisation que vous pouvez consulter en ligne.

https://apropos.erudit.org/fr/usagers/politique-dutilisation/ 


\section{UN PLAGIAIRE ILLUSTRE: FRANÇOIS PERRIN DU LAC*}

L'histoire de tous les pays d'Amérique, à la fin du dix-huitième siècle, est en grande partie dominée par des préoccupations d'ordre économique. Des pays jeunes, dont les structures politiques récentes évoluent rapidement, sont à la recherch t'un équilibre qui doit nécessairement s'appuyer sur une certaine stabilité économique.

Le facteur qui joua le plus grand rôle dans la pénétration de la partie occidentale du continent nord-américain fut assurément le commerce des fourrures. La région des Grands Lacs, très active sous le régime français, reste encore, à cette époque, le point de mire des nations rivales. Le mouvement d'expansion des Etats-Unis vers l'Ouest, surtout après 1783, mérite d'attirer d'abord l'attention. Mais les traiteurs américains ont l'occasion de constater à peu près partout sur le continent l'activité débordante de leurs concurrents anglais et canadiens. Il faut enfin signaler le rôle des Espagnols, maîtres d'une partie de la Louisiane et installés à Saint-Louis des Illinois, près de la confluence du Mississipi et de son plus important affluent, le Missouri.

Les explorations fameuses des capitaines Lewis et Clark, qui atteignirent l'océan Pacifique en 1805, font souvent oublier des prédécesseurs moins illustres, mais aussi importants, qui préparèrent les voies.

Le mieux connu de ces prédécesseurs est peut-être François Perrin du Lac dont le récit de Voyage dans les deux Louisianes et chez les nations sauvages du Missouri, par les Etats-Unis, l'Ohio et les Provinces qui le bordent, en 1801, 1802 et 1803; avec un apperçu des mours, des usages, du caractère et des coutumes religieuses et civiles des Peuples de ces diverses contrées fut publié à Paris et à Lyon,

* Travail présenté à la dernière réunion générale de l'Institut d'Histoire de l'Amérique française. (avril 1953). 
simultanément, dès $1805^{1}$. On accorde habituellement beaucoup d'autorité à ce voyageur dont le récit abonde en détails géographiques, ethnographiques et historiques sur les régions du Haut-Missouri et sur les populations indigènes qui y habitaient à cette époque. Tous les historiens et tous les ethnologues font donc beaucoup de cas de ce Perrin du Lac, et à bon droit. Il serait temps cependant, croyonsnous, de ramener à de plus justes proportions une réputation qui doit la plus grande partie de sa gloire à des emprunts plus que généreux faits dans les notes manuscrites de contemporains moins illustres, dont les récits sont encore ignorés du public, au moins dans des parties fort substantielles.

\section{Qui est Perrin du Lac?}

Et tout d'abord, qui est ce François Perrin du Lac? Aucun historien, à notre connaissance, n'a encore tenté de répondre à cette question qui paraît pourtant fondamentale. C'est pourtant une règle élémentaire en histoire qu'il faut connaître l'auteur de tout témoignage que l'on veut utiliser. A propos de Perrin du Lac, nous n'avons pas trouvé une seule note biographique, et cela dans une cinquantaine d'ouvrages importants (américains surtout) que nous avons consultés. Nous avouons d'ailleurs que nos recherches personnelles sur ce sujet n'ont encore rien donné de bien réconfortant. Perrin du Lac dédie son livre à un certain Monsieur Fourrier, préfet du département de l'Isère. La lettre-dédicace placée en tête du livre se présente ainsi:

\section{Monsieur,}

Un séjour de quelques années chez des peuples nouvellement policés ou sauvages, n'intéressera peut-être que foiblement un savant, un voyageur, à qui la France devra bientôt la connoissance d'un pays que l'on regarde avee raison comme le berceau des Sciences et des Arts. Aussi, n'est-ce ni au savant ni au voyageur que je présente mon Ouvrage; j'en fais hommage au Magistrat chêri, au protecteur généreux qui m'a accueilli et traité comme un ami. S'il y trouve quelques

1. A Paris, chez Capelle et Renaud; à Lyon, chez Bruiset aîné et Buynand. An XIII, 1805. $x-479$ p. $20 \mathrm{~cm}$. Carte. 
moyens de délassement, s'il y puise quelques vérités utiles à la société que ses talens l'appellent à gouverner, j'aurai atteint mon but. J'aurai réussi au-delà de mes espérances, s'il daigne se souvenir toujours que l'Auteur le lui a offert comme un gage de sa reconnoissance, et un monument de son éternel attachement.

Perrin du Lac. ${ }^{2}$

Perrin du Lac semble donc connaître intimement ce préfet de l'Isère. Nous savons par ailleurs que notre voyageur est l'auteur d'une Description du département de l'Isère publiée à Grenoble en $1806^{3}$. Tous ces faits nous incitent à croire que Perrin du Lac était peutêtre originaire de la région de l'Isère, peut-être de Grenoble. Nous espérons pouvoir le vérifier bientôt.

\section{Buts du voyage de Perrin du LaC}

Nous sommes, heureusement, un peu mieux renseignés sur les buts du voyage de Perrin du Lac.

En 1801, les Louisianes venaient d'être cédées à la France. C'est pourquoi, écrit Perrin du Lac, un voyage dans ces régions "devroit présenter un grand intérêt à tous les hommes qui regardent le commerce comme le fondement des états et la base de leur prospérité $^{4}$ ". Notre voyageur s'explique même sur les raisons qui l'incitèrent à traverser d'abord les Etats-Unis: "Je pensai, écrit-il, que pour former un vaste établissement, le point principal étoit de connoître ses voisins et de s'assurer des ressources qu'ils pouvoient lui présenter. Leur langage même me sembla nécessaire pour parcourir des contrées où il étoit presque universellement répandu; d'ailleurs la guerre rendoit difficile et périlleux le passage à la NouvelleOrléans ${ }^{5}$."

2. Voyages dans les deux Louisianes..., p. aiij et suivante.

3. Le Catalogue général des livres imprimés de la Bibliothèque Nationale (Paris) tome CXXXIV, p. 123, mentionne un seul autre ouvrage de Perrin du Lac; il s'agit d'une Description générale du département de l'I sère (Grenoble, J. Allier, 1806. 2 vol. in $8^{\circ}$ ). L'ouvrage est incomplet et n'a apparemment pas été terminé. Cette note nous porte à croire que Perrin du Lac était peut-être originaire de la région de l'Isère.

4. Voyages dans les deux Louisianes..., p. i.

5. Ibid., p. i. 
Perrin du Lac partit de Royan, près de Bordeaux, le 15 mai 1801, à bord d'un navire de la flotte marchande des Etats-Unis. Mais, à cause des tempêtes et des vents, on n'arriva à New York que vers la fin de septembre. La visite des villes et des Etats américains fut une suite à peu près ininterrompue d'émerveillements pour notre voyageur. Il s'écrie. "Nulle part la liberté individuelle n'est autant respectét ${ }^{6} . . . "$ Et plus loin il ajoute: "Dans cet heureux pays, rien ne gêne les ressorts de l'industrie; aucune branche du commerce n'est entravée, aucun droit onéreux ne met de bornes aux élans du génie: quel séjour pour l'homme philosophe et religieux!" Le récit de Perrin du Lac foisonne des détails les plus savoureux sur les provinces maritimes de la Nouvelle-Angleterre, celles de la HauteLouisiane et de l'Ohio. Mais nous nous bornerons ici à la partie d'ailleurs la plus élaborée de ce récit, celle qui traite de la région du Haut-Missouri.

\section{Visite des RÉGIONS dU HAUT-Missouri}

La visite du Haut-Missouri se fit au cours de l'été 1802. On partit de Saint-Louis des Illinois le 18 mai, avec un équipage de dix hommes et toute la marchandise nécessaire pour plusieurs mois de traite. Observons que l'auteur n'a consacré qu'un chapitre à ce que l'on pourrait appeler proprement son "journal". Le reste, c'est-àdire douze chapitres dont quelques-uns sont très longs, constitue en vérité une sorte de traité méthodique et fort documenté sur les diverses tribus indiennes qui habitaient le Haut-Missouri à cette époque: les Ricaras, les Mahas, les Poncas, les Osages, les Kansas, les Sioux et plusieurs autres groupes. Notons que le voyage n'a duré en tout que quatre mois. Comme, par ailleurs, la distance parcourue est de l'ordre de deux mille milles, on peut aisément croire qu'il fut difficile à Perrin du Lac de faire lui-même toutes les observations qu'il rapporte dans son copieux récit.

Il se trouve d'ailleurs une page assez mystérieuse dans le récit de François Perrin du Lac. La voici:

6. Ibid., p. ij.

7. Ibid., p. ij. 
Curieux tout à la fois de connoître par moi-même ces nations non civilisées (du Haut Missouri) et leur manière de traiter avec les Blancs; je chargeai une longue pirogue de tout ce qui pouvoit leur être utile et pris à mon service un ancien traiteur de la rivière des Illinois, qui avoit des connoissances supérieures à celles qu'ont ordinairement les gens de cette espèce. Employé autrefois par la Compagnie du haut Missouri, il avoit remonté cette rivière plus haut qu'aucun autre dans le pays, et avoit passé plusieurs années au milieu de ces peuples, dont il avoit appris les divers dialectes. Il m'a communiqué, sur leurs mœurs, leurs usages et leurs cérémonies, des observations curieuses; et c'est à lui que je dois en grande partie les détails que je donnerai bientôt.

Ce fut pendant son séjour dans les postes les plus éloignés, que le roi d'Espagne promit des récompenses aux traiteurs qui lui donneroient, sur des nations jusqu'alors inconnues, les renseignemens les plus intéressans. Il redoubla d'activité et de travail pour mériter la confiance des autorités supérieures, présenta des mémoires d'une utilité réelle; mais ses peines ainsi que celles de tous ceux qui l'avoient imité, furent entièrement perdues. Ce gouvernement n'en a profité ni pour l'amélioration de son commerce, ni pour l'instruction publique. Tous ces mémoires sont restés dans les archives, dont je les ai tirés moi-même pour en extraire les parties les plus essentielles. Peutêtre cet extrait servira-t-il un jour au gouvernement François, à son commerce, ou à l'homme qui aime à lire dans le grand livre de la Nature ${ }^{8}$.

\section{UNE HYPOTHÈSE INGÉNIEUSE}

Quel est donc ce mystérieux "ancien traiteur de la rivière des Illinois" dont Perrin du Lac dit avoir appris plusieurs détails sur les Indiens de la région du Missouri? De nombreux voyageurs ont parcouru ces régions vers la fin du dix-huitième siècle. Qu'il suffise de nommer, outre Perrin du Lac, les Canadiens Jean-Baptiste Trudeau (ou Truteau) et Pierre-Antoine Tabeau, l'Écossais James

8. Ibid., p. 196-197. C'est nous qui soulignons.

9. Sur ces voyageurs et plusieurs autres de la même époque, voir l'ouvrage récent de A. P. Nasatir, Before Lewis and Clark. Documents illustrating the History of the Missouri, 1785-1804. 2 vol. St. Louis, St. Louis Historical Documents Foundation, 1952. 
Mackay et le Français Victor Collot. Et nous mentionnons seulement ceux dont les notes de voyages sont connues et ont été publiées.

Plusieurs historiens et archivistes se sont spécialisés dans l'étude de ces divers récits. Dans un article de la Mississippi Valley Historical Review ${ }^{10}$, Madame Annie Heloise Abel en vint à émettre l'hypothèse que ce compagnon de Perrin du Lac pouvait fort bien être le Canadien Jean-Baptiste Trudeau. Dans les récits des deux voyageurs, Madame Abel avait en effet trouvé des détails identiques; elle avait constaté que les distances rapportées étaient toujours à peu près les mêmes; elle avait même noté que l'épellation des noms géographiques et de la plupart des noms indiens était la même. Cette hypothèse est parfaitement just fiée et nous sommes en mesure de le démontrer d'une façon que nous estimons définitive, grâce à de récentes découvertes archivistiques.

\section{Les voyages de Jean-Baptiste Trudeau}

Jean-Baptiste Trudeau était né à Montréal le 11 décembre $1748^{11}$. On croit généralement qu'il se rendit à Saint-Louis des Illinois quelques années seulement après la conquête, peut-être dès 1768 , et qu'il y devint le premier maître d'école ${ }^{12}$. Notons toutefois que dans un recensement militaire de 1780, "Baptista Trudo" (forme hispanisée du nom), qui se dit âgé de trente ans et originaire du Canada, déclare le métier de rameur ${ }^{13}$. Les documents du temps reconnaissent qu'il était un homme "prudent, intelligent et expérimente' ${ }^{14}$."

Saint-Louis des Illinois, dont la fondation par Pierre Laclede ne remontait qu'à 1764 , jouait déjà à cette époque un rôle très important dans la Louisiane espagnole. Un lieutenant-gouverneur y

10. Vol. VIII, p. 149-179.

11. Tanguay, Dictionnaire génélogique..., VII: 377.

12. South Dakota Historical Collections, VII: 404 et notes 9 a 16.

13. Louis Houck, The Spanish regime in Missouri (Chicago, 1909, 2 vol.), 1: 188.

14. Rapport du lieutenant-gouverneur espagnol Zénon Trudeau dans Houck, op. cit., II: 253 . 
résidait en permanence et devait s'occuper tout spécialement des relations avec les Indiens du Haut-Missouri. La population totale - blancs, mulâtres et nègres, libres et esclaves - atteignait le millier. Nous croyons que près du quart de cette population était d'origine canadienne ${ }^{15}$.

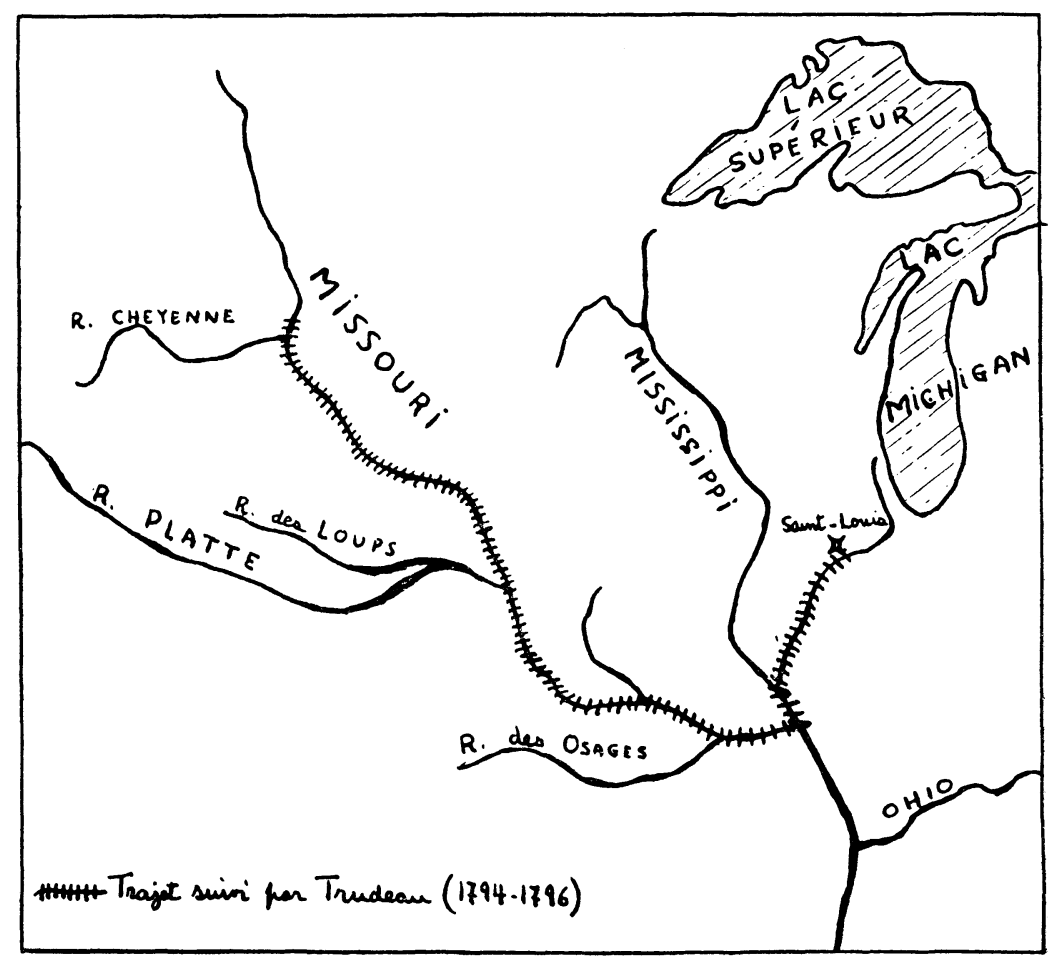

LOCALISATION DES PRINCIPALES RIVIERES DE LA REGION DU HAUT-MISSOURI

(d'après Atlas of the History and Geography of the United States, pl. 39)

$\mathrm{Au}$ cours du mois de mai 1794, un groupe de marchands de Saint-Louis formait, sous les auspices du gouvernement espagnol, une "Compagnie de Commerce pour la découverte des Nations du haut

15. Un recensement de 1796 est rapporté dans Houck, op. cit., II: 141-143. 
Missouri". Des Canadiens figurent parmi les principaux actionnaires de cette compagnie dont les privilèges exclusifs s'étendaient au delà du village des Poncas, près des montagnes Rocheuses, c'est-à-dire dans des régions que les Blancs ne fréquentaient pas assidûment. La Compagnie n'eut pas grands succès. Elle organisa cependant trois expéditions; la première, d'ailleurs la plus importante, fut confiée à Jean-Baptiste Trudeau. Ce choix s'explique sans doute à cause de l'honnêteté, mais aussi de l'expérience considérable de Trudeau qui avait souvent voyagé sur le Missouri et connaissait parfaitement plusieurs dialectes indigènes.

Les Instructions de Trudeau sont datées du 30 juin $1794^{16}$; elles sont signées par les directeurs de la Compagnie, Santiago [ Jacques ] Clamorgan et Antonio Reyhle. La signature de Juan Bautista Truteau (sic) est suivie de celle du lieutenant-gouverneur espagnol de Saint-Louis des Illinois, Zénon Trudeau, lointain parent de son homonyme. Les Instructions comprennent 53 articles dont le dixième et le quarante-deuxième mentionnent que le responsable de l'expédition devra rédiger un journal quotidien qui servira ensuite à la Compagnie. De l'été 1794 au moins de juin 1796, Trudeau rédigea donc un journal dont quelques parties sont connues et ont déjà été publiées. Une première partie se trouve maintenant dans les archives espagnoles de Séville et couvre la période du 7 juin 1794 au 25 mars 1795; le texte original français en a été édité dans l'A merican Historical Review, janvier 1914. La seconde partie du journal se trouve dans les papiers de Thomas Jefferson, à Washington, et couvre la période du 24 mai 1795 au 20 juillet de la même année; des traductions anglaises en ont été maintes fois publiées ${ }^{17}$. C'est en se fondant sur ces parties publiées des récits de Trudeau que Madame Abel a pu émettre l'hypothèse judicieuse à laquelle nous faisions tout à l'heure allusion.

16. Le texte de ces Instructions se trouve dans Houck, op. cit., II: 164-172.

17. Les principales éditions partielles du récit de Trudeau se trouvent dans South Dakota Historical Collections, VIII: 403-433, en traduction anglaise; dans Missouri Historical Society Collections, vol. IV (1912-1913): 9-48, en traduction anglaise; et dans American Historical Review, vol. XIX (octobre 1913 juillet 1914): 299-333, texte original français. L'ouvrage le plus récent à consulter sur Trudeau et sur le problème plus général des expéditions vers l'Ouest américain est celui de A. P. Nasatir, Before Lewis and Clark (cf. note 9). 


\section{Une aUtre version des Journaux de Trudeau}

Il se trouve cependant que les Archives du Séminaire de Québec possèdent, dans le fonds Viger-Verreau, un "Extrait des journaux de $J$. Bte trudeau sur le haut missouris. adressés a Messrs les directeurs de la Compagnie; a St Louis des Illinois ${ }^{18}$ ". Ce document s'avère du plus haut intérêt puisqu'il est à la fois plus détaillé que les versions connues et plus complet, car le journal ne se termine qu'avec le retour de l'expédition à Saint-Louis, le 4 juin 1796. Cette copie du journal est probablement celle que Trudeau avait gardée pour luimême: on y relève en effet de nombreuses ratures et des fautes de toutes sortes qui semblent indiquer une rédaction de premier jet et peu soignée; au contraire, les versions de Séville et de Washington, tout en contenant un nombre encore respectable de fautes, sont tout de même plus soignées et renferment moins de longueurs dans les descriptions et les récits. Ce document mérite, croyons-nous, d'être édité à cause de son intérêt géographique et ethnographique; il est aussi une excellente illustration du rôle important qu'ont joué les Canadiens dans la pénétration de l'Ouest américain. Ce ne sera d'ailleurs pas la seule pièce à être publiée, car la découverte du journal de Trudeau s'est accompagnée d'une autre découverte encore plus sensationnelle.

\section{Trudeau, auteur D'Une description des Indiens DU HaUt-Missouri}

Jean-Baptiste Trudeau a en effet rédigé un autre document de très grande importance, à la demande du lieutenant-gouverneur espagnol Zénon Trudeau lui-même. Il s'agit donc d'un texte qui diffère complètement du journal et intitulé "Description abrégée du Haut-Missouri adressée à Monsieur Don Zénon Trudeau, lieutenantgouverneur de la partie occidentalle des Illinois, \&tc. par J B Trudeau,

18. Archives du Séminaire de Québec, Boîte 10, numéro 74. 187 pages manuscrites, $13 \times 81 / 2$ pouces.

19. Archives du Séminaire de Québec, Boîte 10, numéros 70 (70 p.), 71 (38p.), 72 (22 p.), 73 ( 35 p. non paginées). $13 \times 8 \frac{1}{2}$ pouces. 
voyageur ${ }^{19}$ ". Voici d'ailleurs en quels termes le voyageur canadien s'exprime dans la lettre qui sert d'introduction à sa "Description": $:^{20}$

Monsieur ${ }^{21}$,

Je n'aurois osé entreprendre une ouvrage qui est hors de mes forces, ny vous la prếsenter Si vous neussiez paru le désirer de moi dans la lettre que vous m'avez fait l'honneur de m'écrire; en datte du trois de may. mil Sept Cent quatre vingt quinze lorsque Jétois Chez Ces peuples lointains. Jhésiterois encore de le faire Si je ne connoissois votre indulgence, et que je ne fus persuadé que vous ne ferez point attention au peu d'élégance qui regne dans Cet ouvrage et au manque des belles expressions et des termes recherchés, que vous ny rencontrerez point; J'espère que vous vous Souviendrez en le Lisant que l'autheur bien loin d'être un académicien; n'est qu'un pauvre voyageur qui quoiqu'il ait reçu dans Sa Jeunesse une certaine éducation, L'a si fort négligé depuis qu'un Stile fleuri et embelli de toutes les grâces de la réthorique N'est plus de Son usage. Je ne puis vous présenter qu'un ouvrage, dicté dans le langage le plus Simple et le plus familier; Mais je vous proteste que tout y est Conforme a la vérité, Car jai observé dans ces mémoires de ne rien marquer qui soye[ nt ] faux et incroyabl [ e ], Comme vous me L'avez prescrit dans votre Lettre.

J'ai fait un rapport exacte, dans mes journaux que vous avez lù, des assemblées, Conseils, et parlement que j'ai tenu avec les nations arricaras, Chaguiennes et Siouses, au nom de Sa majesté Catholique; auxquelles vous avez envoyé par moy, pavillons, lettres patentes, et présents. j'ai aussi envoyé des paroles a toutes les nations Circonvoisines, aux mandannes, aux gros ventres, aux Cayowas, aux tocaminanbiches, aux pitapahato, et tokiwako, Dont plusieurs Chefs sont venus me voir dans le Cour de l'étée que je passai au village (des) arricaras, pour les maintenir en paix et en union; et par là faciliter les entreprises de commerce et de découverte, que la nouvelle Compagnie du haut Missouris Se proposoit dy faire Sous ma gestion. Les presents que vous leurs avez envoyez par moy, et les paroles que vous leurs avez adressées au nom du roy, ont été reçu de tous Ces peuples. Situés à l'occident de Cette rivierre, avec les plus grandes marques de Joie et d'applaudissement. J'ai fait réunir avec les arricaras

20. Archives du Séminaire de Québec, 19: 70, p. 1 et 2.

21. Don Zénon Trudeau, lieutenant-gouverneur à Saint-Louis des Illinois. 
et les Chaguiennes quatre gros villages de Sioux qui faisaient une Cruelle guerre au premier ${ }^{22}$.

La "Description abrégée du Haut-Missouri" est un long document qui se trouve également dans le fonds Viger-Verreau des Archives du Séminaire de Québec. C'est en le comparant avec le récit de Perrin du Lac que nous avons pu vérifier l'exactitude de l'hypothèse de Madame Abel. A n'en point douter, Jean-Baptiste Trudeau fut le compagnon de route de Perrin du Lac. Mais l'étude comparative des récits des deux voyageurs permet d'affirmer bien autre chose. Dans la suite de son voyage, Perrin du Lac a, comme il le dit luimême, découvert les écrits de Trudeau dans les Archives de la Nouvelle-Orléans. Les "Voyages dans les deux Louisianes..." ne sont qu'une reprise des excellentes notes de Trudeau; mais Perrin du Lac passe pour être l'auteur de ces notes descriptives du Haut-Missouri: il s'agit en réalité d'un plagiat authentique.

Perrin du Lac cache le véritable auteur de ces notes qu'il avoue cependant avoir utilisées (cf. plus haut). Drôle d'utilisation! Il s'agit en effet d'une copie intégrale dont le seul mérite est d'adapter le texte en le présentant dans un meilleur français et en éliminant certains détails mineurs: mince mérite, en vérité! Le plan des chapitres est le même. Les idées sont les mêmes. L'articulation de la pensée est identique. Les distances rapportées sont les mêmes - 150 milles est quelquefois remplacé par 50 lieues! Perrin du Lac se dit témoin de faits qui se sont vraiment passés six ou sept ans auparavant; il n'a même pas pris soin de modifier la chronologie. Il s'agit donc d'un plagiat à la fois dans l'esprit et dans la lettre. Les pièces que nous citons en appendices le démontrent abondamment. Perrin du Lac ne s'est apparemment pas douté un seul instant qu'on pourrait découvrir un jour ce qui semble avoir été la copie personnelle de l' "ancien traiteur des Illinois", Jean-Baptiste Trudeau ${ }^{23}$.

Fernand Grenier

22. Suit le "Commencement" de la "Description abrégée..."

23. Les Voyages de Jean-Baptiste Trudeau sur le Haut-Missouri, de $1794 \grave{a}$ 1796, édités par Fernand Grenier et Fernand Ouellet, paraîtront au cours de 1953 aux Presses Universitaires Laval et formeront le tome III des Publications des Archives du Seminaire de Quebec. 


\section{APPENDICE I}

Jean-Baptiste Trudeau

Table des chapitres se rapportant au Haut Missouri

I - Description abrégée du Haut-Missouri

p. 2-40 (du manuscrit)

II - Origine, croyance, cérémonies des Sauvages dans le culte qu'ils rendent à la divinité

$41-61$

III - Mœurs des Sauvages du Haut-Missouri $\quad 62-82$

IV - Habit, logement, complexion des Sauvages du Haut-Missouri

V - Amours et mariages des Sauvages du HautMissouri

$11-28$

VI - Manière de faire la paix, d'aller en calumet et de le danser

$$
1-10^{*}
$$

VII - La danse du calumet

11-23

VIII - La danse du soleil

24-28

IX - La danse du bœuf

28-30

X - Manière incivile et grossière des Sauvages

$31-33$

XI - Guerres des Sauvages

* Le texte est copié dans 4 cahiers grand format. La pagination recommence dans chacun des cahiers. 
François Perrin du LaC

Table des chapitres se rapportant au Haut Missouri

XXIX - Peuples sauvages du haut Missouri

p. $257-264$

XXX - Croyance des Sauvages; leur culte; leurs cérémonies religieuses; leurs usages à la mort d'un parent; comment il sont enterrés.

$265-276$

XXXI - Mœurs des Sauvages du Haut Missouri; exemple de justice exercée par les Sioux sur un de leurs grands chefs.

$277-286$

XXXII - Manière de vivre intérieure des Sauvages; occupations des femmes.

$287-292$

XXXIII - Guerre des Sauvages; manière de la faire; retour d'une parti vainqueur; danse des Chevelures; excès de leur tristesse après une défaite; traits de courage et de sang-froid.

292-313

XXXIV - Paix des Sauvages; comment ils vont en calumet; réception des guerriers envoyés en calumet.

$314-321$

XXXV - Danse du Calumet.

$322-329$

XXXVI - Danse du Soleil.

$330-333$

XXXVII - Danse du Bœuf.

334-335

XXXVIII - Habillement, logement, complexion et tempérament des Sauvages du haut Missouri. Leurs armes. 336-346

XXXIX - Amours et Mariage des Sauvages.

$347-354$

XL - Réflexion sur la vie des Sauvages: comparaison avec celle de l'homme vivant en société. 
APPENDICE II

\section{QUelques EXEMPles DE PASSAGes PLAGIÉS}

\section{TRUDEAU}

La grande nation des Padas qui parcourrent les bords de la rivierre platte n'est éloignée du Missouris a l'endroit du territoire des aricaras que de dix jours de marche de guerriers qui peuvent être évaluée a soixante, et quatre vingt de nos lieues. Les halitannes ou têtes pelées peuples errants occupent tout le pays au delà de la rivière platte jusques sur les bords de la rivierre des arkansas. et s'étendent le long des grandes montagnes qui Séparent le nouveau mexique de cette partie de lamérique méridionale... (p. $19 \mathrm{du}$ Ms., ASQ, V-V, 10: 70)

Dans tous les villages du haut missouris il y a une grande Cabane Qu'ils appellent La loge des vieux, qui ne sert a dautres usages qu'aux festins, aux danses, Et a toutes les Cérémonies de leur culte superstitieus; C'est là aussi ou ils recoivent les Etrangers Et tiennent les Conseils de la Nation, elle est encore la loge de grâce, Cest a dire que le plus grand ennemi qui pourroit Entrer dans cette Cabane Sans être au paravant arrété, auroit la vie sauve... (p. 42 du Ms., ASQ, $\mathrm{V}-\mathrm{V}, 10: 70)$

Tous ces peuples sauvages ne connoissent point de subordination

\section{PERRIN DU LAC}

La grande nation des Padaws qui parcourent la rivière Plate, n'est éloignée de celle des Ricaras que d'environ dix journées de chasseur, que l'on peut estimer à vingt-cinq milles chacune. Les Halisanes ou Têtes pelées sont errans, chassent sur l'autre rive de la rivière Plate, jusques sur les bords de celle des Arkansas, et s'étendent aux pieds des montagnes du nouveau Mexique dans un territoire abondant en toutes sortes d'animaux... (p. 161)

Dans tous les villages du haut Missouri, il y a une grande cabane appelée la Loge des vieillards, qui est consacrée aux festins, aux danses et aux cérémonies religieuses. C'est là qu'ils donnent audience aux étrangers, et qu'ils délibèrent sur les intérêts de la nation. On la nomme aussi la Loge de grace, parce que si leur plus cruel ennemi pouvoit y pénétrer, non-seulement il ne perdroit point la vie, mais il seroit à l'abri de toute espèce d'insulte... (p. 269270)

Tous les peuples sauvages ne connaissent ni distinction ni su- 
ni de distinction entre eux; ils vivent dans une espèce d'égalité conforme aux sentiments de la nature; ils n'ont ni loix, ni juges, ni prêtres, et ne s'embarassent point de cette multitude de loix ne de cette foule de juges d'avocats et de notaires, qui quoique sagement établis pour le maintien et le bon ordre de nos sociétés en sont très souvent, les tyrans, par le mauvais usage qu'ils font des lois dont ils sont les dépositaires... (p. $53 \mathrm{du}$ Ms., ASQ, V-V, 10: 70)

Tous les hommes, jeunes et vieux des peuples qui habitent le haut Missouri sont également nus comme la main dans la saison d'été, les uns et les autres portent négligeamment sur leurs épaules une couverture de peau de biche ou de vache passée très légère. Les jeunes hommes les ont garnies de pores-épies, ou mattachés de diverses couleurs... (p. $1 \mathrm{du}$ Ms., ASQ, V-V, 10: 71).

Les Sauvages a qui On racontent nos mariages ne peuvent pas concevoir que les hommes Blanes qui ont tant desprit, Et de Connoissance Soient assez aveugles ou ignorants pour ne pas Connoitre que le mariage Est pour eux une Source de chagrin Et de peine, ils Regardent comme une chose monstrueuse de Se lier L'un alautre Sans espérance de pouvoir jamais rompre Ce nœud. Enfin de quelques bonnes raisons qu'on puisse les- bordination. Ils n'ont aucun chef civil ni militaire, et vivent, quoiqu'en société, dans l'égalité la plus parfaite, sans lois, sans juges et sans prêtres... (p. 277)

Parmi tous les peuples qui habitent le haut Missouri, tous les hommes, jeunes ou vieux, sont nus pendant l'été. Une couverture de peau de biche passée, qu'ils portent négligeamment sur leurs épaules (à peu près comme les Espagnols portent le manteau) leur sert plutôt de parure que de vêtement. Celles des jeunes gens sont garnies de porc-épies ou matachées de diverses couleurs... (p. 336)

Une des choses que dans nos mœurs les Sauvages conçoivent le plus difficilement, e'est le lien indissoluble que nous contractons avec nos femmes. Comment, disent-ils, ces hommes Blanes si supérieurs à tous les autres par leur génie, leur industrie et leur connoissances, peuvent-ils former des nœuds qu'ils ne doivent jamais rompre? Esclaves de leurs femmes, ils sont obligés d'en supporter les caprices ou la mauvaise hu- 
presser ils Se tiennent fermes Et immobiles adire que nous sommes les esclaves des femmes... (p. 11 du Ms., ASQ, V-V, 10-71) meur, et se condamnent à vivre avec elles, quelque dégoût qu'elles puissent leur inspirer. Aucune raison ne peut les faire revenir sur cette opinion: ils finissent par dire que celui qui se lie perd sa liberté, et cesse par-là même d'être homme... (p. 350-351)

\section{APPENDICE III}

\section{LA DANSE DU BOEUF}

\section{TRUDEAU}

Cette danse se pratique entre différentes nations alliez qui Seront Campées auprès l'une de l'autre; ou chez un même peuple qui sera séparé en différents villages voisins, dont L'un ira donner le divertissement de Cette danse à Lautre; ils Sassemblent dans une des plus grandes Cabannes $\mathrm{du}$ village quinze a vingt hommes des plus braves de la nation vetus de leurs habillements de guerre, leurs armes armes a la main portant chacun un bonnet fait de hurre de bouf qui descend Sur les épaules auquel est attaché de chaque Coté une Corne tel que Ces animaux en portent. Le devant de Ces bonnets est orné d'une bande de cuire large de trois doigts garni de porte épic ou de rassade bleu; il n'y a que Les hommes braves qui ont droit de porter Ces sortes de bonnets tout autre qui le porteroit qui n'auroit pas fait aucun Coup remarquable Seroit moqué. Ils sortent de Cette Cabanne accompagné des chantres, des timbales (et des chichicois leurs instruments ordinaires), et Savançent en dansant ou plutôt

\section{PERRIN DU LAC}

Lorsque deux nations amies sont campées à peu de distance, ou que la même nation est divisée en plusieurs villages peu éloignés, elles se donnent souvent le plaisir de cette danse, pour laquelle il n'y a ni saison ni jour marqués. Un village veut-il danser le bruf à ses voisins, quinze ou vingt guerriers recommandables s'assemblent dans une cabane, revêtent leurs habits de guerre, et s'arment de toutes pièces. Ils se coiffent d'un bonnet fait de la dépouille d'une tête de bœuf sauvage, à laquelle les cornes sont attachées; et le tout est lié avec une lisière de cuir, garnie de porc-épic, soigneusement travaillée.

Dans cet accoutrement, les guerriers s'avancent jusqu'au lieu destiné à la danse, accompagnés des chanteurs qui portent à la main les chichakois et les timbales. Ils s'étudient à contrefaire l'animal qu'ils cherchent à imiter; tantôt ils mugissent comme lui, et font voler au loin la poussière avec leurs pieds; tantôt ils se poursuivent les uns les autres, ainsi que font ces animaux du temps du 
en Sautant jusqu'auprès des habitations de Ceux a qui ils vont donner le divertissement; a Chaque pose en Marchant, il Contrefont les bœufs, faisant voler la poussièrre en l'air avec leurs pieds, meuglant et Se poursuivant tel que Ces animeaux le pratiquent dans la saison de leur rut étant entrés Soit dans la grande cabanne des vieux, Soit sur la place au milieu $\mathrm{du}$ village ils y dansent des demi jours entiers, observant toujours les memes gestes et Contortions. Pendant que la danse dure les vieillards ne cessent d'haranguer partout le village de faire des présents aux danseurs $\mathrm{Ce}$ qui ne manque pas; Car ces gens là S'en retournent presque toujours chez eux chargés de butin qui leur a été jetté, ou fait présent, qu'ils partagent ensuitte entre eux également. (ASQ, V-V, 10: 72, p. 25-27 du Ms.) rut; tantôt ils imitent les combats qu'ils se livrent à coups de cornes et de pieds. Ils continuent ces jeux en variant leurs attitudes et leurs gestes, en présence de ceux à qui ils donnent ce divertissement, pendant une demi-journée, et quelquefois davantage, sans laisser appercevoir le moindre signe de fatigue. Ils font au contraire avant de les cesser de nouveaux efforts d'activité. Pendant la danse, les vieillards ne cessent de haranguer leurs gens et de les exciter à faire des présens aux danseurs: ces présens sont presque toujours si considérables, qu'ils peuvent en distribuer à toutes leurs familles et à leurs amis. (p. 334-335).

\section{LES É T U DES \\ de l'Institut d'Histoire de l'Amérique française}

Abonnez-vous à ces Etudes. L'Institut d'Histoire ne publie que des oeuvres d'une valeur scientifique incontestée.

Déja parues :

Iroquoisie, de M. Léo-Paul Desrosiers (\$2.25)

François Bigot, administrateur français, de M. Guy Frégault -2 vol. $-(\$ 4.50)$

Louis Jolliet - Vie et voyages, du Père Jean Delanglez, s.j. ( $\$ 2.50)$

Le Grand Marquis - Pierre de Rigaud de Vaudreuil et La Louisiane, de M. Guy Frégault - (\$3.50)

On peut s'abonner aux Etudes de l'Institut en faisant un dépôt de \$10. au siège social de l'Institut: 261 , ave Bloomfield, Outremont 8, P.Q. 